\title{
Assessment of Thermomechanical Couplings in Tribological Surface Transformations: Application to the Irreversible Near-Surface Solid-Solid Phase Transformations
}

\author{
Grégory Antoni \\ Haute Ecole d'ingénierie et de Gestion du Canton de Vaud, Institut COMATEC, Route de Cheseaux 1,
} 1401 Yverdon-les-Bains, Switzerland

Correspondence should be addressed to Grégory Antoni; antoni.gregory@yahoo.fr

Received 27 November 2012; Accepted 27 January 2013

Academic Editors: V. E. Buchanan, Y. Desplanques, Z. Wen, H. Xing, and Y. Zhang

Copyright (c) 2013 Grégory Antoni. This is an open access article distributed under the Creative Commons Attribution License, which permits unrestricted use, distribution, and reproduction in any medium, provided the original work is properly cited.

Tribological Surface Transformations (TSTs), which are irreversible near-surface solid-solid phase transformations, tend to occur on railroads frequented by heavy freight trains. The present study is proposed to assess the contribution of thermomechanical coupling processes to the emergence and development of TSTs near the surface of the rails.

\section{Introduction}

The irreversible quasi-surface solid-solid phase transformations known as Tribological Surface Transformations (TSTs), which occur near the surface of rails [1-3], are induced by contact fatigue processes $[4,5]$, occurring after the passing of several trains on the rail tread. In order to account for the emergence and development of TSTs, it was suggested in a previous study [6] that TSTs result from repeated normal and stress loads, combined with highly localized thermal effects caused by friction between the wheels and rails. It is proposed here to test this assumption, namely that the thermomechanical coupling processes may generate TSTs near the surface of the rails.

\section{A Thermodynamic Framework for Modelling Irreversible Near-Surface Solid-Solid Phase Transformations}

In this section, the constitutive equations involved in the thermomechanical model for irreversible solid-solid phase transformations [6] are recalled:

$$
\begin{aligned}
& \dot{\boldsymbol{\epsilon}}=\dot{\boldsymbol{\epsilon}}^{\mathrm{te}}+\dot{\boldsymbol{\epsilon}}^{\mathrm{pc}}+\dot{\boldsymbol{\epsilon}}^{\mathrm{pz}}, \\
& \dot{\boldsymbol{\epsilon}}^{m}=\frac{1}{3} \operatorname{Tr}\left(\dot{\boldsymbol{\epsilon}}^{m}\right) \mathbf{G}+\dot{\boldsymbol{e}}^{m} \quad \text { with } m=(\text { te, } \mathrm{pc}, \mathrm{pz}),
\end{aligned}
$$

$$
\begin{aligned}
\dot{\boldsymbol{\sigma}}= & -\dot{P} \mathbf{G}+\dot{\mathbf{s}}, \\
\dot{P}= & -\frac{(3 \lambda+2 \mu)}{3}\left[\operatorname{Tr}(\dot{\boldsymbol{\epsilon}})+\frac{\dot{z}}{\kappa}-3 \alpha \dot{T}\right], \\
\dot{\mathbf{s}}= & 2 \mu\left(\dot{\mathbf{e}}-\dot{\mathbf{e}}^{\mathrm{pz}}-\dot{\mathbf{e}}^{\mathrm{pc}}\right), \\
\dot{\boldsymbol{\epsilon}}^{\mathrm{te}}= & \lambda \operatorname{Tr}\left(\dot{\boldsymbol{\epsilon}}^{e}\right)+2 \mu \dot{\boldsymbol{\epsilon}}^{e}+\alpha \dot{T} \mathbf{G}, \\
\dot{\boldsymbol{\epsilon}}^{\mathrm{pz}}= & -\frac{1}{3} \frac{\dot{z}}{\kappa} \mathbf{G}+\dot{p} \frac{3}{2 \sigma_{\mathrm{eq}}} \mathbf{s}, \\
\dot{z}= & \kappa \dot{p}, \\
\dot{p}= & \frac{\langle 1-z\rangle}{\eta}\left\langle\frac{T}{T_{i}^{z}}-\exp \left(-\frac{\langle P\rangle}{\omega}\right)\right\rangle \\
& \times H\left(P+\delta\left[(1-z) T_{i}^{z}-T\right]\right), \\
\dot{\boldsymbol{\epsilon}}^{\mathrm{pc}}= & \dot{\mathrm{e}}^{\mathrm{pc}}=\dot{v} \frac{3}{2 \sigma_{\mathrm{eq}}} \mathbf{s}, \\
\dot{v}= & \frac{\left\langle\sigma_{\mathrm{eq}}-\left(\sigma_{y}+h v\right)\right\rangle}{\xi \sigma_{y}},
\end{aligned}
$$

where $T$ is the absolute temperature, $\boldsymbol{\epsilon}=1 / 3 \operatorname{Tr}(\boldsymbol{\epsilon}) \mathbf{G}+$ $\mathbf{e}$ is the small-strain tensor, $\boldsymbol{\epsilon}^{\boldsymbol{e}}$ is the elastic strain tensor, 
$\boldsymbol{\epsilon}^{m}=1 / 3 \operatorname{Tr}\left(\boldsymbol{\epsilon}^{m}\right) \mathbf{G}+\mathbf{e}^{m}$ (where $m=($ te, $\mathrm{pc}, \mathrm{pz}$ )) are the thermoelastic, classical (visco-)plastic and TRIP-like strain tensors, respectively, $\mathbf{G}$ denotes the metric tensor, $v$ denotes the isotropic hardening variable associated with classical (visco-)plasticity, $\mu>0$ and $\lambda>-(2 / 3) \mu$ are the Lamé constants, $\alpha>0$ is the thermal expansion coefficient, $z=$ $\rho_{d} / \rho \in[0,1]$ is the mass fraction of the daughter phase (where $\rho_{d}=\rho_{\text {martensite }}$ is partial mass density of the daughter phase, $\rho=\rho_{d}+\rho_{p}$ is total mass density, and $\rho_{p}=\rho_{\text {ferrite }}$ is partial mass density of the parent phase (i.e., the ferrite phase)), $\kappa>0$ is a material parameter characterizing the change in the density occurring during phase transformations, $T_{i}^{z}$ is the solid-solid phase transformation temperature when the pressure is zero, $\omega>0$ is a material parameter characterizing a "sensitive pressure" level, $h>0$ is a material parameter characterizing the linear isotropic hardening associated with classical plasticity, $\delta \geq 0$ is a material parameter associated with the latent heat of the phase transformation (see [7]), $\boldsymbol{\sigma}$ is the Cauchy stress tensor, $-P \mathbf{G}=1 / 3 \operatorname{Tr}(\sigma) \mathbf{G}$ and $\mathbf{s}$ are the spherical and deviatoric parts of the Cauchy stress tensor, respectively, $\sigma_{\mathrm{eq}}=(3 / 2 \mathbf{s}: \mathbf{s})^{1 / 2}$ is the Von Mises equivalent stress, $\sigma_{y}$ is the classical yield strength, $\xi$ and $\eta$ are the characteristic time of the viscous effects associated with TRIP-like and the classical plasticity processes, respectively, $\langle\cdot\rangle$ denotes the Macaulay brackets $(\langle x\rangle=x$ when $x \geq 0$ and $\langle x\rangle=0$ when $x<0)$, and $H(\cdot)$ denotes the Heaviside step function $(H(x)=1$ when $x \geq 0$ and $H(x)=0$ when $x<0)$.

Based on the assumption that TSTs are induced by "strong" thermomechanical coupling-the combined effects of the temperature and mechanical processes-the heat equation can be written as follows (see [7]), taking $\dot{T}$ to denote the increase in the temperature:

$$
\begin{aligned}
\rho C_{\epsilon} \frac{T}{T_{i}} \dot{T} & -k \Delta T \\
= & -(3 \lambda+2 \mu) \alpha T \operatorname{Tr}(\dot{\boldsymbol{\epsilon}}) \\
& +\mathbf{s}:\left(\dot{\mathbf{e}}^{\mathrm{pc}}+\dot{\mathbf{e}}^{\mathrm{pz}}\right)-h v \dot{v} \\
& +\frac{1}{\kappa}\left[P-(3 \lambda+2 \mu) \alpha T+\delta(1-z) T_{i}^{z}\right] \dot{z}
\end{aligned}
$$

where $C_{\epsilon}>0$ is the specific heat capacity, $T_{i}$ is the initial temperature, $k$ is the thermal conductivity, and $\Delta$ denotes the Laplacian. The mass density $\rho$ is roughly an equal value to the initial mass density, $\rho \simeq \rho_{i}$. The parameters, $\rho, C_{\epsilon}, k, \lambda, \mu$, and $\alpha$ are not assumed to depend on the temperature and are taken to be identical in both phases.

Note that the thermodynamic consistency of this thermomechanical model was checked in our previous study [6].

\section{Numerical Results and Discussion}

3.1. Heat Equation: Source Terms. In this Section, it is proposed to assess the effects of the "source" terms on the evolution of the temperature on the emergence and development of TSTs. In our previous study [6], the source terms corresponding to the thermoelasticity, the classical (visco-)plasticity, and the irreversible solid-solid phase transformations were omitted in the first approximation, and in the second approximation, we dealt only with the steady case of the heat equation, that is, $\Delta T$.

However, the orders of magnitude of these terms can be assessed, a posteriori, and compared. In the incremental form, the full heat equation (2) can written as follows:

$$
\begin{aligned}
\rho C_{\boldsymbol{\epsilon}} & \frac{T_{n}}{T_{i}} \frac{T_{n+1}-T_{n}}{\Delta t_{n}}-k \Delta T \\
= & \underbrace{-(3 \lambda+2 \mu) \alpha T_{n} \operatorname{Tr}\left(\left(\frac{\boldsymbol{\epsilon}_{n+1}-\boldsymbol{\epsilon}_{n}}{\Delta t_{n}}\right)\right)}_{\text {term due to the thermoelasticity }\left(\Phi_{\mathrm{te}}\right)} \\
+ & \underbrace{\mathbf{s}:\left(\frac{\mathbf{e}_{n+1}^{\mathrm{pc}}-\mathbf{e}_{n}^{\mathrm{pc}}}{\Delta t_{n}}\right)-h v\left(\frac{v_{n+1}-v_{n}}{\Delta t_{n}}\right)}_{\text {term due to the classical (visco-)plasticity }\left(\Phi_{\mathrm{pc}}\right)} \\
+ & \underbrace{\mathbf{s}:\left(\frac{\mathbf{e}_{n+1}^{\mathrm{pz}}-\mathbf{e}_{n}^{\mathrm{pz}}}{\Delta t_{n}}\right)+\frac{1}{\kappa}\left(P_{n}-(3 \lambda+2 \mu) \alpha T_{n}+\delta\left(1-z_{n}\right) T_{i}^{z}\right)\left(\frac{z_{n+1}-z_{n}}{\Delta t_{n}}\right)}_{\text {term due to the irreversible solid-solid phase transformation }\left(\Phi_{\mathrm{pz}}\right)},
\end{aligned}
$$

where $(\cdot)_{n+1}$ (resp. $\left.(\cdot)_{n}\right)$ denote the quantities at time $t_{n+1}$ (resp. $t_{n}$ ) and $\Delta t_{n}=t_{n+1}-t_{n}$ is the time step.

Although these source terms $\left(\Phi_{\mathrm{te}}, \Phi_{\mathrm{pc}}\right.$ and $\left.\Phi_{\mathrm{pz}}\right)$ were obtained from a temperature field (see [6]) resulting from the resolution of the heat equation in the steady case, the results obtained in the previous study [6] can be used to assess, in a first approach, the heat sources at any time, as defined by the term on the right-hand side of (3). Note that the physical interpretations associated with each parameter of (3), including their chosen set value, are discussed in [7].

Figure 1 shows the heat field sources at time $t=10 \mathrm{~s}$ under the thermomechanical loading conditions studied in [6]. Note that the heat sources thus obtained are much greater than the power of the internal stresses (Figure 2). The heat source caused by the thermoelastic processes alone is plotted in Figure 3. In fact, in the volume measuring $25 \mathrm{~mm}^{3}(10 \mathrm{~mm}$ $\times 2.5 \mathrm{~mm} \times 1 \mathrm{~mm})$ in the immediate vicinity of the surface where the thermomechanical loading is applied, the power dissipation of the heat sources caused by the thermoelastic and classical (visco-)plastic processes and the irreversible solid-solid phase transformation coupling is approximately $425.10^{-3} \mathrm{~W}$, whereas the power of the internal stresses is only of $145.10^{-3} \mathrm{~W}$. On the other hand, the power dissipation of the heat source due the thermoelastic effects alone is approximately $402.10^{-3} \mathrm{~W}$. It can be seen here that under the boundary conditions defined in [6], the thermoelastic effects are nonnegligible. It is now proposed to assess these thermoelastic coupling effects in the case of a unidimensionnal problem (Section 3.2).

3.2. Thermoelastic Coupling: 1-D Problem. In order to estimate the thermoelastic coupling effects, it is proposed to take a 1-D thermoelastic problem, that of a bar in steel (occupying a domain $\Omega \in[0, L] \subset \Re)$ exposed to thermomechanical loads (Figure 4), assuming the presence of small perturbations. 


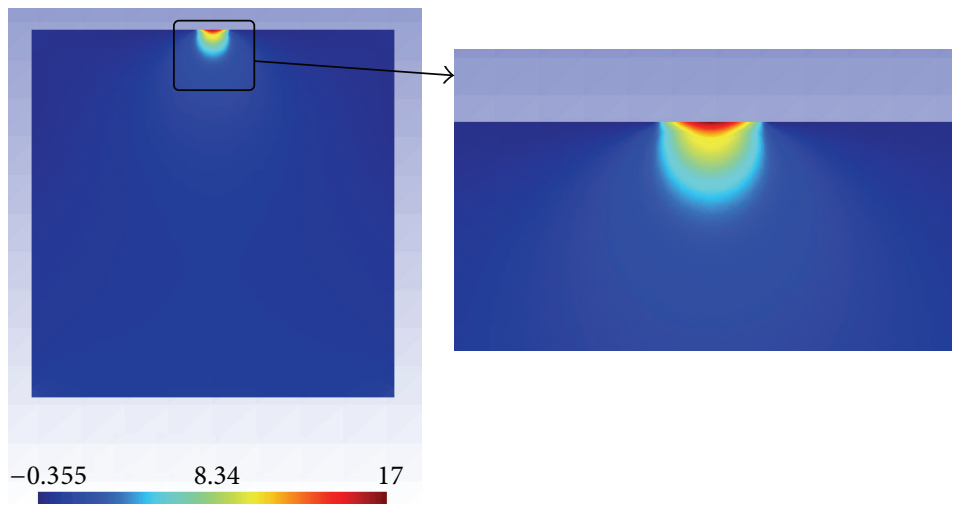

Figure 1: Heat sources due to the thermoelastic and classical (visco-)plastic processes and the irreversible solid-solid phase transformations (unit: $\mathrm{MPa} \cdot \mathrm{s}^{-1}$ or $\mathrm{mW} \cdot \mathrm{mm}^{-3}$ ) at time $t=10 \mathrm{~s}$ under the previously defined thermomechanical loading conditions [6], where $T_{i}^{z}=1000 \mathrm{~K}$, $\omega=900 \mathrm{MPa}, \eta=10^{3} \mathrm{~s}$, and $\kappa=10^{2}$.

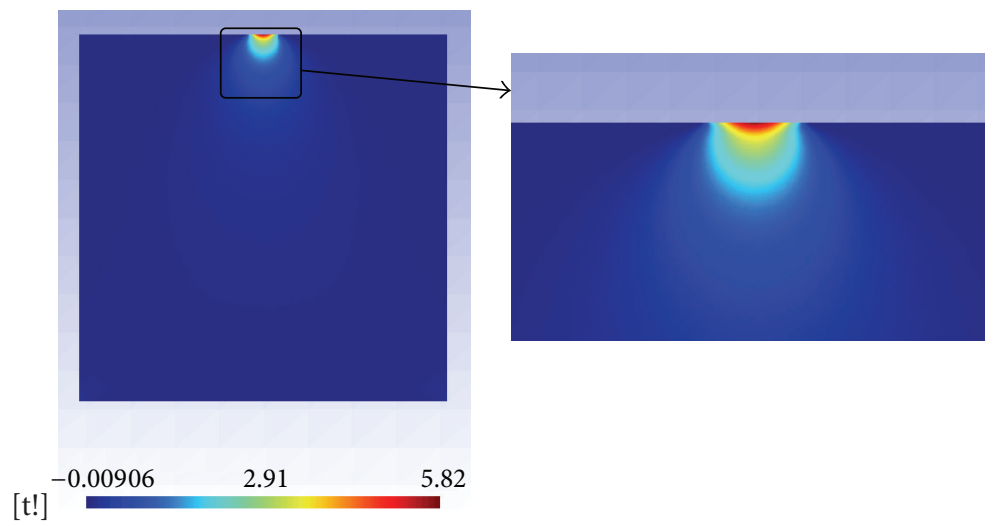

Figure 2: Power of the internal stresses (unit: $\mathrm{MPa} \cdot \mathrm{s}^{-1} \mathrm{or} \mathrm{mW} \cdot \mathrm{mm}^{-3}$ ) at time $t=10 \mathrm{~s}$ under the previously defined thermomechanical loading conditions [6], where $T_{i}^{z}=1000 \mathrm{~K}, \omega=900 \mathrm{MPa}, \eta=10^{3} \mathrm{~s}$, and $\kappa=10^{2}$.

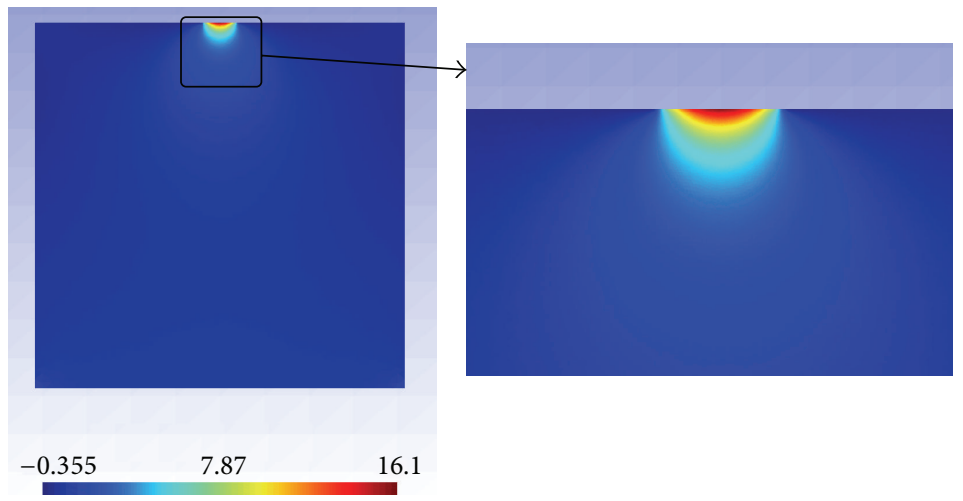

Figure 3: Heat source due to the thermoelastic processes alone (unit: $\mathrm{MPa} \cdot \mathrm{s}^{-1} \mathrm{or} \mathrm{mW} \cdot \mathrm{mm}^{-3}$ ) at time $t=10 \mathrm{~s}$ under the previously defined thermomechanical loading conditions [6], where $T_{i}^{z}=1000 \mathrm{~K}, \omega=900 \mathrm{MPa}, \eta=10^{3} \mathrm{~s}$, and $\kappa=10^{2}$. 
The Helmholtz free energy potential per unit mass $\psi$ of the material considered (see [8]) can be written as follows:

$$
\begin{aligned}
\psi(T, \epsilon)= & \frac{E}{\rho_{i}}\left[\epsilon-\alpha\left(T-T_{i}\right)\right]^{2} \\
& -\left[\frac{C_{\epsilon}}{2 T_{i}}+\frac{E \alpha^{2}}{2 \rho_{i}}\right]\left(T-T_{i}\right)^{2}+\psi_{i}, \quad \forall x \in \Omega,
\end{aligned}
$$

where $T$ and $\epsilon$ denote the absolute temperature and the smallstrain, respectively, $\rho_{i}$ is the initial mass density, $E$ is the Young's modulus, $C_{\epsilon}>0$ is the specific heat capacity, $T_{i}$ is the initial temperature, $\alpha$ is the thermal expansion coefficient, and $\psi_{i}$ is the initial Helmholtz free energy potential per unit mass.

H1: the mass density in the actual $(\rho)$ and initial $\left(\rho_{i}\right)$ configurations is approximately equal, that is, $\rho \simeq \rho_{i}$.

Assuming $H 1$, the state functions associated with both the temperature and the strain variable are:

$$
\begin{gathered}
S=-\frac{\partial \psi}{\partial T}=C_{\epsilon} \frac{\left(T-T_{i}\right)}{T_{i}}+\frac{E}{\rho_{i}} \alpha \epsilon, \quad \forall x \in \Omega, \\
\sigma=\rho \frac{\partial \psi}{\partial \epsilon} \simeq \rho_{i} \frac{\partial \psi}{\partial \epsilon}=E\left[\epsilon-\alpha\left(T-T_{i}\right)\right], \quad \forall x \in \Omega,
\end{gathered}
$$

where $S$ and $\sigma$ denote the specific entropy per unit mass and the Cauchy stress.

The local expression of First Principle of Thermodynamics (FPT) is:

$$
\rho \dot{e}=\sigma \epsilon-\operatorname{div}(\mathbf{q})+r \Longleftrightarrow \rho T \dot{S}+\operatorname{div}(\mathbf{q})-r=0, \quad \forall x \in \Omega,
$$

where $e=\psi+$ TS denotes the internal energy potential per unit mass and $\mathbf{q}$ is the heat flux vector. Note that the remote heat production rate will be omitted from now on, that is, $r=$ 0 .

H2: Fourier's law is adopted:

$$
\mathbf{q}=-k \nabla T, \quad \forall x \in \Omega,
$$

where $\mathbf{q}$ denotes the heat flux vector.

Combining $H 1, H 2$, and (5), the FPT can be written as follows (also called "Heat Equation"):

$$
\rho_{i} C_{\epsilon} \frac{T}{T_{i}} \dot{T}-k \Delta T=-E \alpha T \dot{\epsilon}, \quad \forall x \in \Omega .
$$

H3: the absolute temperature in the actual $(T)$ and initial $\left(T_{i}\right)$ configurations are fairly similar, that is, $T / T_{i} \cong 1$.

Using (6) and based on $H 2$ and $H 3$, (9) can be written as follows:

$$
\rho_{i} C_{\epsilon} \dot{T}-k \Delta T=-E \alpha T_{i}\left[\frac{\dot{\sigma}}{E}+\alpha \dot{T}\right], \quad \forall x \in \Omega .
$$

H4: the term " $\rho_{i} C_{\epsilon}$ " is very large in comparison with " $E \alpha^{2} T_{i}$ " (because $\alpha \lll 1$ ), that is, $\rho_{i} C_{\epsilon} \ggg E \alpha^{2} T_{i}$.

The Heat Equation (10) obtained under the assumption $H 4$, neglecting the convective terms of the particular derivative and taking the lateral thermal leaks in the bar into account, can be rewritten in the following form:

$$
\rho_{i} C_{\epsilon}\left[\frac{\partial T}{\partial t}+\frac{\left(T-T_{i}\right)}{\tau}\right]-k \frac{\partial^{2} T}{\partial x^{2}}=-\alpha T_{i} \frac{\partial \sigma}{\partial t}, \quad \forall x \in \Omega,
$$

where $\tau$ denotes the characteristic time of the lateral thermal leaks in the bar.

Assuming that both inertial and gravity effects are nonpresent, the local expression of quasi-static equilibrium can be reduced to:

$$
\frac{\partial \sigma}{\partial x}=0, \quad \forall x \in \Omega .
$$

Based on (12), the stress field $\sigma(x, t)$ is uniform throughout the bar, that is, for all $x \in \Omega, \sigma(x, t)=\sigma_{0}(t)$. The boundary conditions are summarized in Figures 4 and 5, and the constitutive thermomechanical parameters used are:

$$
\begin{aligned}
& C_{\epsilon}=450 \mathrm{~J} \cdot \mathrm{kg}^{-1} \cdot \mathrm{K}^{-1} ; \\
& E=2 \times 10^{5} \mathrm{MPa} ; \quad \rho_{i}=78 \times 10^{-7} \mathrm{~kg} \cdot \mathrm{mm}^{-3} ; \\
& \alpha=12 \times 10^{-6} \mathrm{~K}^{-1} ; \quad k=5 \times 10^{-2} \mathrm{~W} \cdot \mathrm{mm}^{-1} \cdot \mathrm{K}^{-1} .
\end{aligned}
$$

Figure 6(a) gives the temperature distribution in the depth of the bar at time $t=t_{L}=10 \mathrm{~s}$, taking into account (dashed, dotted, and dotted-and-dashed lines) or not (solid lines) the heat sources due to the thermoelastic effects under three different loads $(-200,-500$, or $-1000 \mathrm{MPa})$. It can be seen from this figure that when the source term is zero (solid line), the temperature reaches $T=T_{i}$ at a depth of about $45 \mathrm{~mm} \leq x \leq L$, whereas in the thermoelastic source term (Figure 6(a)), a temperature increase of about $20 \mathrm{~K}$ for $\left|\sigma_{0}^{\min }\right|=200 \mathrm{MPa}$ (dashed line), $35 \mathrm{~K}$ for $\left|\sigma_{0}^{\min }\right|=$ $500 \mathrm{MPa}$ (dotted line), and $55 \mathrm{~K}$ for $\left|\sigma_{0}^{\min }\right|=1000 \mathrm{MPa}$ (dotted-and-dashed line) is observed in the center of the bar. The characteristic time of the lateral thermal leaks, $\tau$, clearly depends on the thermomechanical loading rate: $\tau=30 \mathrm{~s}$ for $\left|\sigma_{0}^{\min }\right|=200 \mathrm{MPa}, \tau=13 \mathrm{~s}$ for $\left|\sigma_{0}^{\min }\right|=500 \mathrm{MPa}$ and $\tau=8 \mathrm{~s}$ for $\left|\sigma_{0}^{\mathrm{min}}\right|=1000 \mathrm{MPa}$. The stress-strain curve at point $x=L / 200$ at time $t=t_{L}=10 \mathrm{~s}$ is plotted in Figure 6(b) only for $\left|\sigma_{0}^{\mathrm{min}}\right|=1000 \mathrm{MPa}$. The total strain at the end of the loading $\left(t=t_{L}\right)$ is about $-4.8 \times 10^{-3}$, and small-strain conditions therefore apply.

The results obtained in this section show that the source term due to the thermoelasticity is responsible for a variably marked increase in the temperature increase near the end of the bar and throughout the bar, but that the thermomechanical loading conditions considered here (see Figure 5) have greater effects in the immediate vicinity of the end of the bar. In line with what was observed in our previous study [6], these terms can therefore be reasonably neglected in the first approximation.

\section{Conclusion}

This paper deals with the contribution of thermomechanical coupling processes to the emergence and development of irreversible near-surface solid-solid phase transformations. Based on the results obtained here, the thermoelastic effects can be said to predominate over the effects of the other thermomechanical heat source resulting from classical (visco-)plasticity and irreversible solid-solid transformations. Although these source terms cause the temperature to 


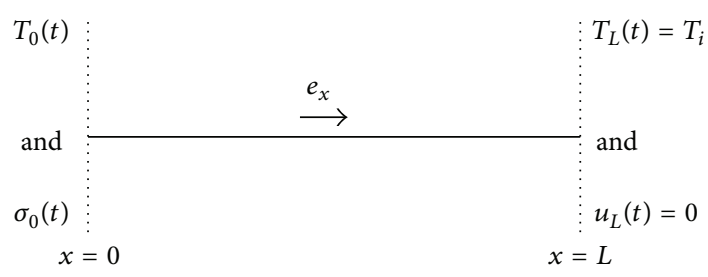

Figure 4: Geometry $\left(L=100 \mathrm{~mm}\right.$ ) and boundary conditions (with $\left.T_{i}=300 \mathrm{~K}\right)$. On the left end of the bar $(x=0)$, a thermomechanical loading $\left\{\sigma_{0}(t), T_{0}(t)\right\}$ is applied whereas both zero displacement, $u_{L}(t)=0$, and an initial set temperature, $T_{L}(t)=T_{i}$, are imposed constant with time on the right end of the bar $(x=L)$, that is, $u_{L}(t)=0$, for all $t$ and $T_{L}(t)=T_{i}$, for all $t$; $\mathbf{e}_{x}$ denotes the unit vector in the $x$-direction.

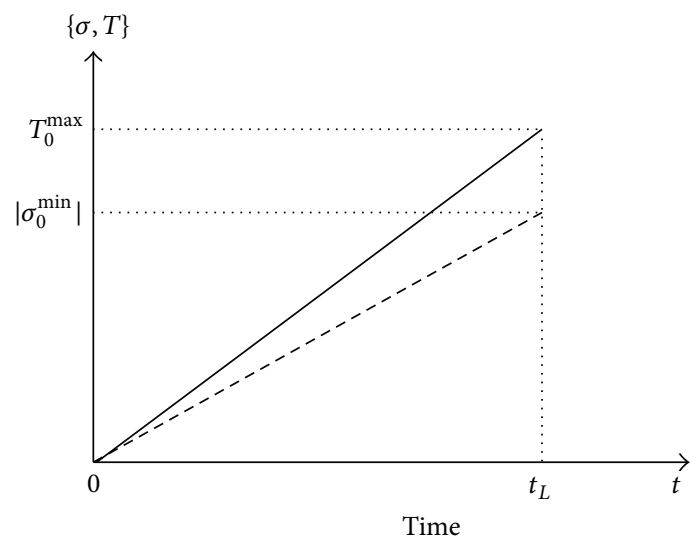

FIGURE 5: Thermomechanical loading applied (on the left end of the bar, $x=0$ ). Note that the function $T_{0}(t)\left(\right.$ resp. $\sigma_{0}(t)$ ), which progresses linearly with time, is given by a solid line with $T_{0}^{\max }=450 \mathrm{~K}$ in time $t_{L}=10 \mathrm{~s}$ (resp. a dashed line with $\sigma_{0}^{\min }$ under three different loads $\{-200,-500,-1000\} \mathrm{MPa})$

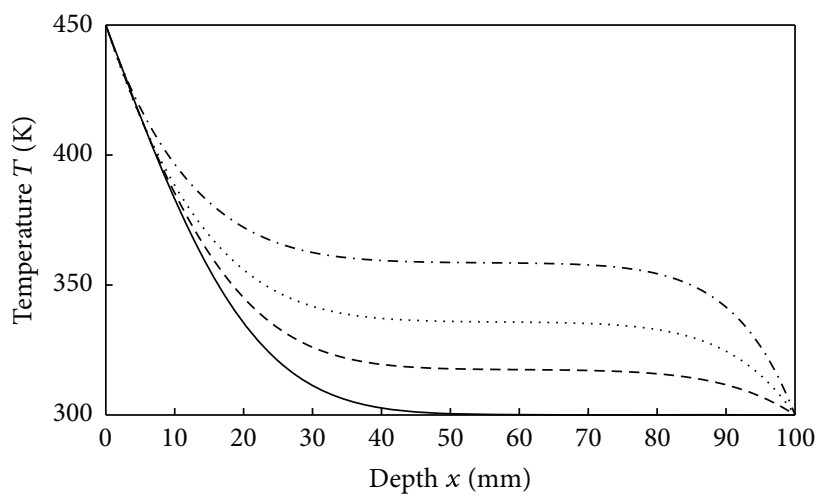

(a)

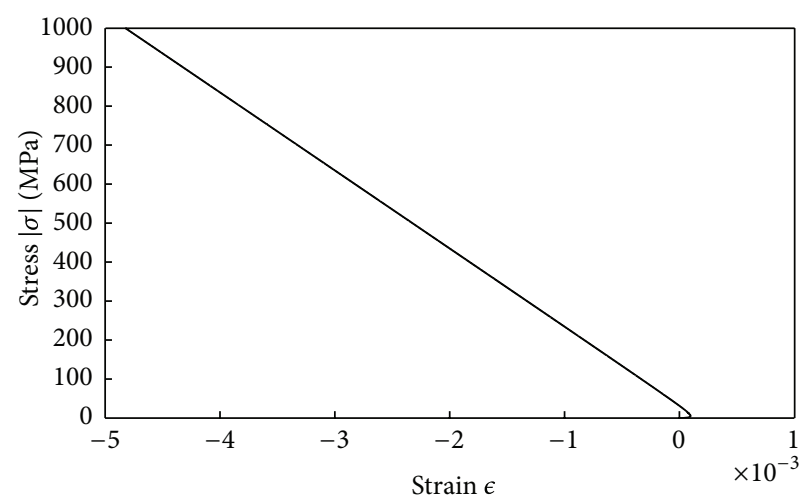

(b)

Figure 6: (a) Temperature distribution in the bar at time $t=t_{L}=10 \mathrm{~s}$ taking into account (dashed, dotted, and dotted-and-dashed lines) or not (solid line) the heat sources due to the thermoelastic effects under three different loads: $\sigma_{0}^{\min }=-200 \mathrm{MPa}$ (dashed line) where $\tau=30 \mathrm{~s}$, $\sigma_{0}^{\min }=-500 \mathrm{MPa}$ (dotted line) where $\tau=13 \mathrm{~s}$, and $\sigma_{0}^{\min }=-1000 \mathrm{MPa}$ (dot-dash line) where $\tau=8 \mathrm{~s}$. (b) Stress-strain curve at point $x=L / 200$ at time $t=t_{L}=10 \mathrm{~s}$ under the loading conditions $\left|\sigma_{0}^{\min }\right|=1000 \mathrm{MPa}$.

increase at the surface and in the depth of the structure, it emerges clearly from this study that the thermomechanical loads applied constitute the main driving force responsible for generating TSTs.

\section{Acknowledgment}

The author is indebted to Dr. Jessica Blanc for her help with this paper. 


\section{References}

[1] G. Baumann, H. J. Fecht, and S. Liebelt, "Formation of whiteetching layers on rail treads," Wear, vol. 191, no. 1-2, pp. 133-140, 1996.

[2] W. Österle, H. Rooch, A. Pyzalla, and L. Wang, "Investigation of white etching layers on rails by optical microscopy, electron microscopy, X-ray and synchroton X-ray diffraction," Materials Science and Engineering A, vol. 303, no. 1-2, pp. 150-157, 2001.

[3] A. Pyzalla, L. Wang, E. Wild, and T. Wroblewski, "Changes in microstructure, texture and residual stresses on the surface of a rail resulting from friction and wear," Wear, vol. 250-251, no. 2 , pp. 901-907, 2001.

[4] R. I. Carroll and J. H. Beynon, "Rolling contact fatigue of white etching layer-part 1: crack morphology," Wear, vol. 262, no. 910, pp. 1253-1266, 2007.

[5] R. I. Carroll and J. H. Beynon, "Rolling contact fatigue of white etching layer-part 2: numerical results," Wear, vol. 262, no. 910, pp. 1267-1273, 2007.

[6] G. Antoni, "Contribution to the Modelling of the Tribological Surface Transformations," ISRN Tribology, vol. 2013, Article ID 254705, 6 pages, 2013.

[7] G. Antoni, Transformations Tribologiques de Surface: Une Approche Thermo-Mécanique [Thèse de doctorat], Université de Provence, Marseille, France, 2010.

[8] J. Lemaitre and J. L. Chaboche, Mécanique des Matériaux Solides, Dunod, Paris, France, 1996. 

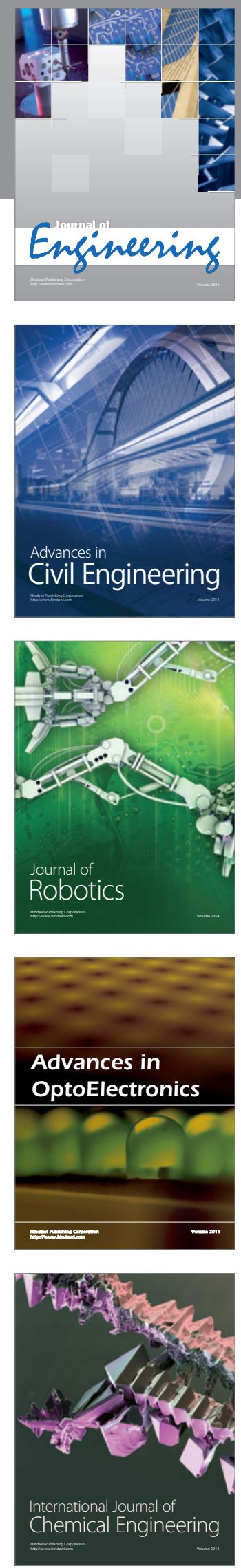

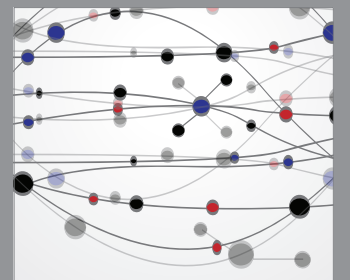

The Scientific World Journal
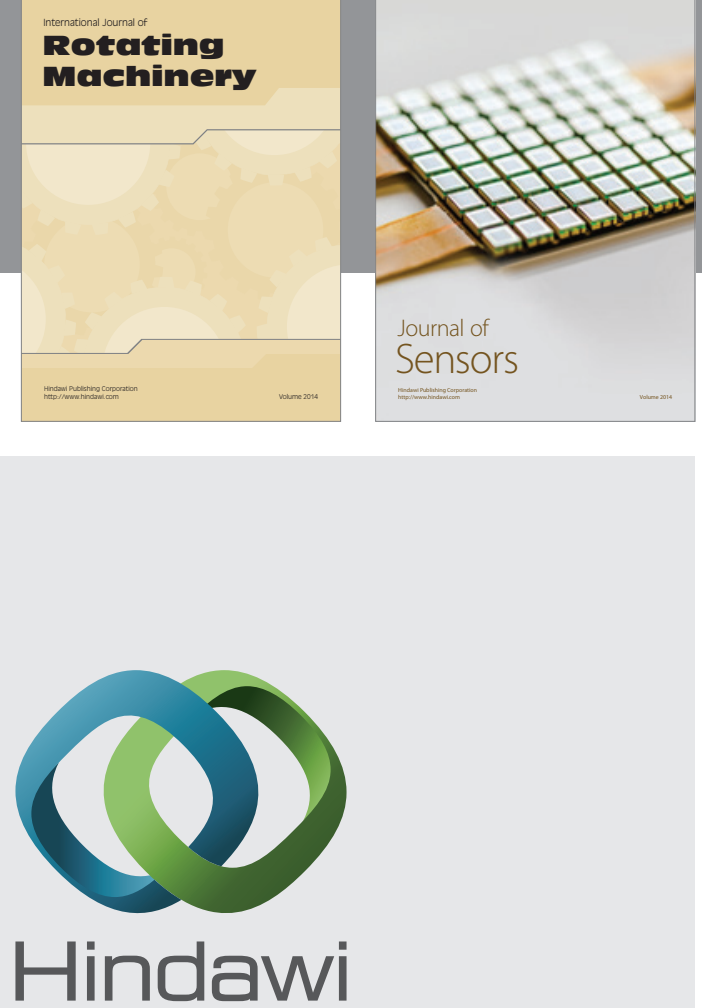

Submit your manuscripts at http://www.hindawi.com
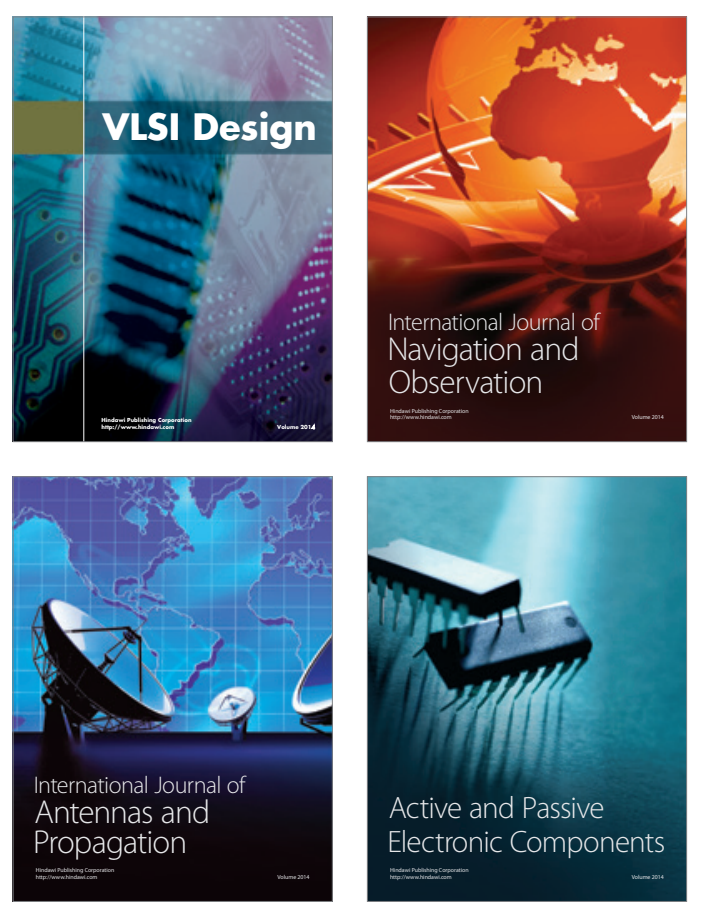
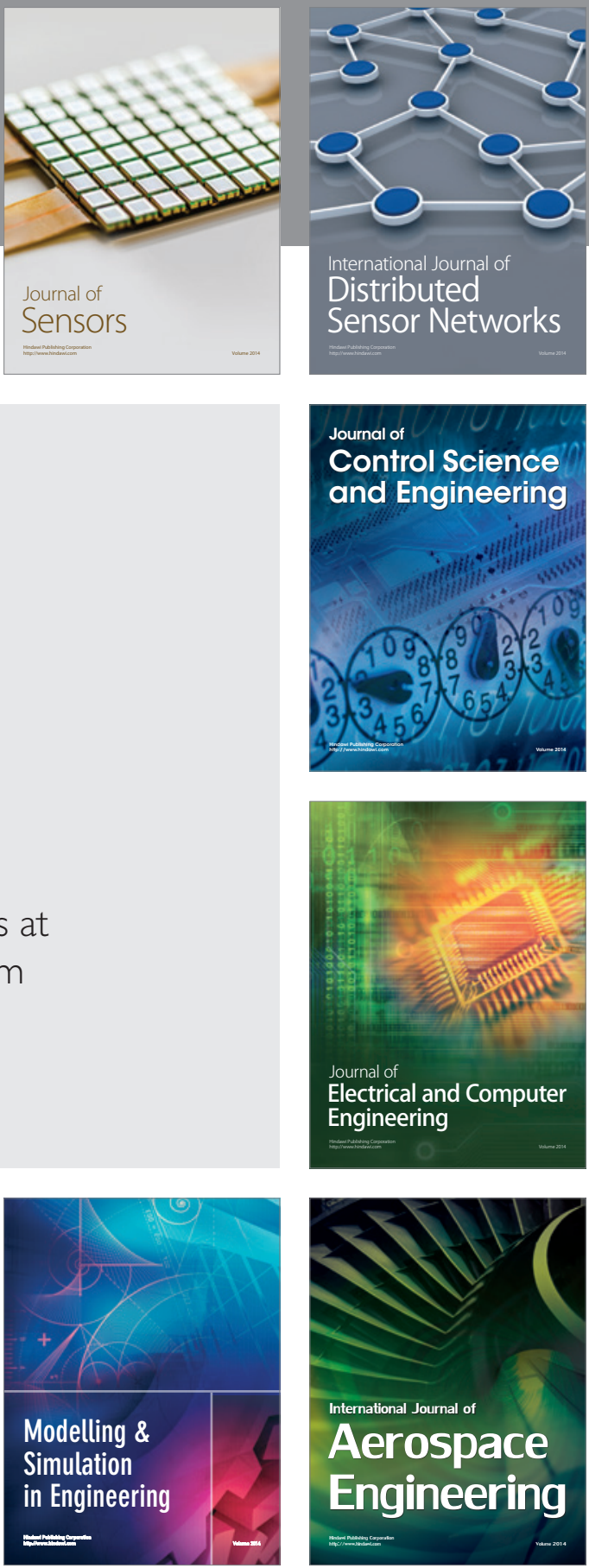

Journal of

Control Science

and Engineering
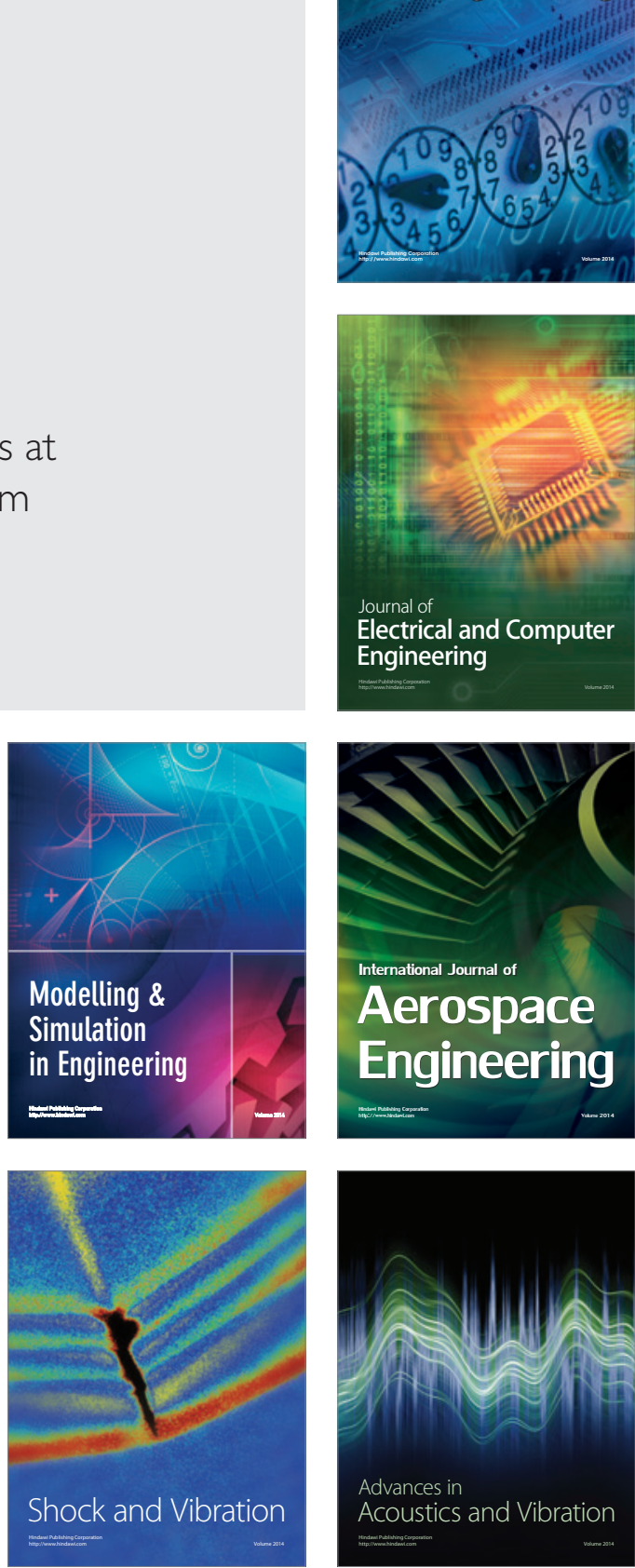